\title{
Analysis of drug-protein interaction in bio-inspired microwells
}

\author{
Himani Sharma ${ }^{1} \cdot$ Ambuja Navalkar $^{2} \cdot$ Samir K. Maji $^{2} \cdot$ Amit Agrawal $^{1}$ \\ (c) Springer Nature Switzerland AG 2019
}

\begin{abstract}
The study of binding of drug with protein is an important process in determining the activity and consequences of the drug. However, the current screening methods to investigate the drug-protein interaction are still pervaded with some disadvantages such as large requirement of sample volume and the need of expensive instrumentation. Here, we present a proof of concept for a method to study the interaction of different kinds of drugs with protein on a microwell array, to enable the understanding of pharmacokinetics in a fast and facile manner. To this end, we demonstrated the applicability of present method by analysing the interaction of fluorescently labelled bovine serum albumin (FITC-BSA) with sulphanilamide (SMO) and sulfamethoxazole (SMZ) drugs on lotus leaf-based microwells. The quenching mechanism of FITC-BSA in the presence of SMO and SMZ is visualized through time-lapse fluorescence imaging for the first time in femto-litre sized microwells. The binding parameters extracted from the present method are found to be in good agreement with the fluorescent spectroscopy measurements. The interaction between protein and drugs was further corroborated by molecular docking analysis and FT-IR technique. Finally, the bio-inspired microwell array was integrated into a magnetic digital microfluidic device to facilitate investigation of drug-protein interaction in power-free and cost-effective manner. A substantial decrease in sample volume and relatively short processing times when compared to other bulk methods makes the present method attractive. The results presented here therefore open up the possibility of rapid measurement of binding parameters in protein-ligand interaction studies in low-resource environments.
\end{abstract}

Keywords Bio-inspired B BSA · Drug-protein interaction · Fluorescence quenching $\cdot$ Microwells

\section{Introduction}

Discovery of micromolecule (drug) typically begins with the identification and validation of target macromolecule (protein) [1]. In pharmacokinetic and pharmacodynamic studies, binding of drugs to plasma proteins is an important factor that influences absorption, distribution, metabolism, and excretion (ADME) properties of the drug [2]. For example, serum albumin is the one of the main carriers in blood, which facilitates the transfer of various drugs to its target. It is a well-known fact that metabolism and distribution of various drugs is affected by the interaction of drug with the protein in blood [3]. Subsequently, understating of drug-protein binding is an essential step and routinely investigated in pre-clinical stages of the drug discovery.

Typically, the methods to investigate drug-protein interaction can be divided into separative and non-separative techniques. The separative techniques such as equilibrium dialysis [4], ultrafiltration [5], ultracentrifugation [6], liquid chromatography [7], and capillary electrophoresis [8] rely on separation of bound drug-protein complex from the unbound protein using permeable/porous structures. On the other hand, the non-separative techniques that include spectroscopy (UV, IR and fluorescence) $[9,10]$, isothermal titration calorimetry $[11]$ and surface plasmon resonance $[12,13]$ are used to detect change in a physiochemical property of either drug or protein due

Amit Agrawal, amit.agrawal@iitb.ac.in | 'Department of Mechanical Engineering, Indian Institute of Technology Bombay, Powai, Mumbai 400076 , India. ${ }^{2}$ Department of Biosciences and Bioengineering, Indian Institute of Technology Bombay, Mumbai 400076 , India. 
to binding. These techniques, though widely used, suffer from several drawbacks such as non-specific adsorption of drug/protein, sensitivity, pressure-mediated volume change, difficulty in protein immobilization and sedimentation. Furthermore, the expensive instrumentation, requirement of large volume of sample, long incubation time and complexity of operations makes these methods laboratory-intensive.

Microfluidics can overcome these limitations, due to its ability to work with reduced sample volume (less than $100 \mu \mathrm{L}$ ), short processing times and rapid analysis, thereby imparting ultra-sensitivity, reduced cost and operational simplicity. For the very reason, a large number of microfluidic devices have been developed for applications related to protein measurement [14], single cell analysis [15], and polymerase chain reaction [16] in the last decade. Recently, Petra et al. [17] employed a microfluidic device to study the interaction between human serum albumin (HSA) and warfarin (WAR) drug. They segmented HSA functionalized magnetic beads and WAR into small microdroplets, and separated the unbound WAR and HSA-WAR complex using a T-junction microchannel. The compartmentalization of drug and protein into microdroplets ensure fast reaction and high-throughput analysis. In a similar vein, microwells can also compartmentalize samples in ultra-low volumes (typically in femtolitres to microliters), and have been exploited in detection and analysis of proteins and single cell studies. Due to the small volume of each microwell, a marked improvement in analytical sensitivity and speed can be achieved. For example, Wang et al. [18] demonstrated capturing and detection of insulin-like growth factor 1 receptor (IGF-1R) in microwells in the attomolar range. Although microwell-based microfluidic platforms have been popular in applications mentioned above, there are no such studies reported hitherto on the investigation of drug-protein interaction.

In this work, we propose to use a microwell array to exploit the benefit of extremely small volume of reaction chambers to capture the fluorescence quenching of drug-protein complex, thereby facilitating determination of ADME properties of the drug. We have taken advantage of the surface topography of a lotus leaf to fabricate microwells on polydimethylsiloxane (PDMS) slabs by using soft lithography process to reduce the cost of fabrication of the microwell platform. Furthermore, as a proof-of-concept we have built a microdevice by integrating the microwell platform based on our previous work, to envisage possibility of automating the proposed method. The bovine serum albumin (BSA) was chosen as a model protein which has versatile binding ability and water solubility. The binding of two drugs viz., sulfanilamide (SMO) ( $p$-Aminobenzenesulfonamide), Sulfamethoxazole (SMZ) (N1-(5-methyl-3-isoxazolyl), with BSA were investigated on our proposed microwell platform. The SMO and SMZ are well-known antibacterial drugs used in treating lower respiratory infections and urinary tract infections [19]. The interaction between BSA and SMO or SMZ has been already established before [20], therefore making it a convenient model to study on microfluidics platform. In our work, we have used this drug-protein interaction to showcase the usability of our low-cost microwell based microdevice. Furthermore, the interaction between SMO and SMZ with fluorescein isothiocyanate labelled-BSA (FITC) was studied quantitatively by FT-IR and molecular docking.

\section{Proposed methodology}

The method proposed in this work aims to capture the quenching of fluorescence signal in the microwell array, resulting from varying the dosage of either SMZ or SMO to the FITC-BSA. To this end, we have employed a PDMS surface containing an array of bio-inspired microwells, placed on a glass slide as shown in Fig. 1a. The microwellsurface was rendered hydrophilicity before the experiments to ensure complete filling of the microwells. Initially, a droplet of $2 \mu \mathrm{L}$ containing FITC-BSA was pipetted on the surface containing empty microwells and allowing FITCBSA to be captured inside microwells using capillary forces as shown in Fig. $1 \mathrm{~b}$. The concentration of FITC-BSA was fixed at $40 \mu \mathrm{M}$ in the experiments. Once the droplet was equilibrated on the microwell-surface, another droplet of $2 \mu \mathrm{L}$ containing either SMZ or SMO was pipetted on the microwell array filled with the FITC-BSA. The dosage of the drugs was varied from 20 to $60 \mu \mathrm{M}$ in the experiments. Subsequently, the time-lapse study of interaction between FITS-BSA and SMO/SMZ was conducted using fluorescent microscopy. The fluorescence signal from the microwells was recorded at different time intervals from 1 to $12 \mathrm{~min}$. The binding interaction of protein and drugs was investigated through the Stern-Volmer analysis. The dynamic quenching constant, binding constant and number of binding sites were evaluated. The interaction on FITC-BSA with $\mathrm{SMO} / \mathrm{SMZ}$ in the microwells was further quantified by FT-IR studies. Finally, the integration of biomimetic microwell to low cost magnetic actuated digital microfluidics device was undertaken.

\section{Materials and methods}

\subsection{Reagents}

Bovine serum albumin (BSA, fraction $\mathrm{V}$, approximately $99 \%$, protease free), SMZ and SMO were obtained from Sigma Aldrich Company, USA. For labelling of BSA with 
A

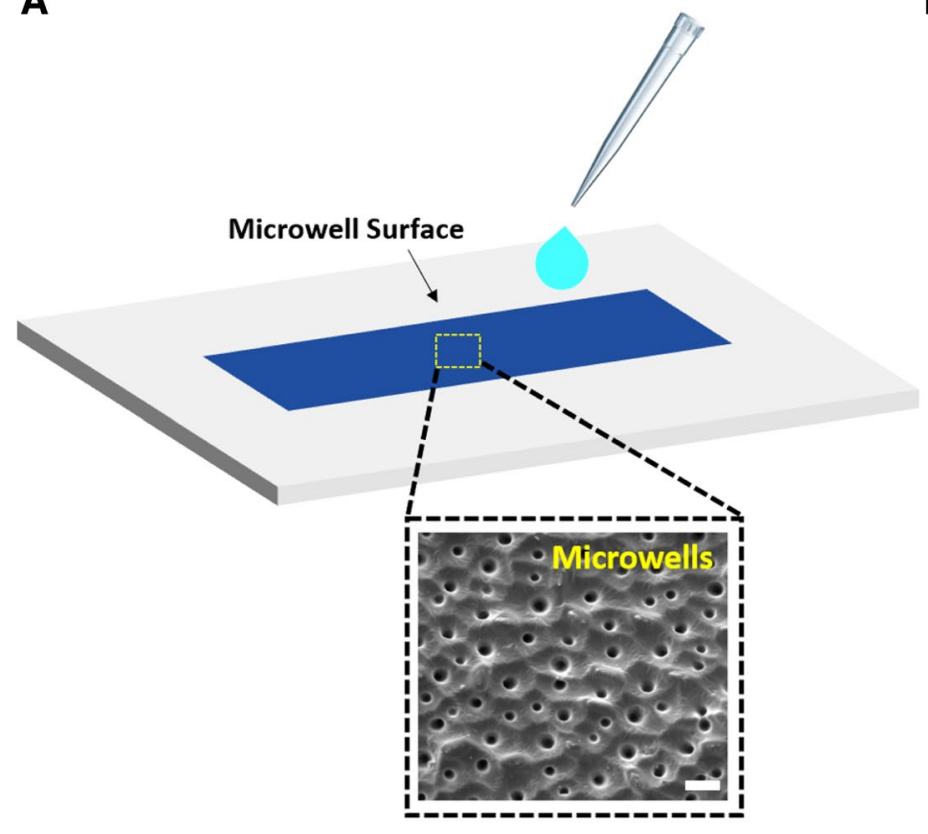

B

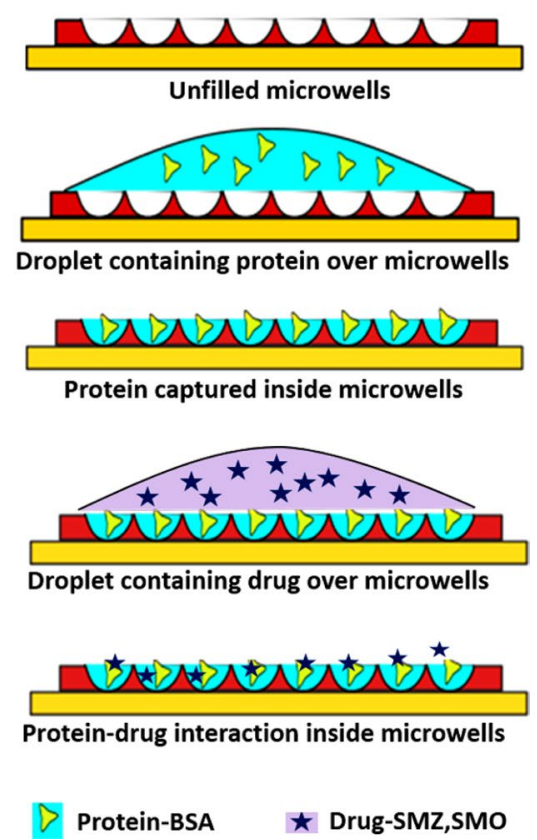

Fig. 1 a Schematic showing droplet containing either drug/protein being pipetted on microwell-surface (scale bar-20 $\mu \mathrm{m}$ ), $\mathbf{b}$ an illustration of sequence of steps of the method to visualize fluorescence quenching of protein in the presence of drug

fluorescein isothiocyanate (FITC, Life Technologies, Thermofisher Scientific, USA), a stock of $5 \mathrm{mg} / \mathrm{mL}$ BSA was prepared in a $0.1 \mathrm{M}$ sodium bicarbonate buffer of, $\mathrm{pH} 9$. In continuation, FITC was dissolved in Dimethyl sulfoxide (DMSO, Thermofisher, USA) at $1 \mathrm{mg} / \mathrm{mL}$. Then, for each $1 \mathrm{~mL}$ of protein solution, $50 \mu \mathrm{L}$ of FITC solution was added in $5 \mathrm{~mL}$ aliquots. After the required amount of FITC solution was added, the reaction was incubated in the dark for $8 \mathrm{~h}$ at $4{ }^{\circ} \mathrm{C}$. Then, a $50 \mathrm{mM} \mathrm{NH}_{4} \mathrm{Cl}$ (Sigma Aldrich) solution was added and incubated for $2 \mathrm{~h}$ at $4{ }^{\circ} \mathrm{C}$. The protein was dialyzed using PBS to remove the excess free dye by using gel filtration with PD-10 column (GE Healthcare, USA). The absorbance of the eluent (at $280 \mathrm{~nm}$ and $495 \mathrm{~nm}$ ) was taken to measure the concentration of the protein and the degree of labelling of BSA was calculated. The $40 \mu \mathrm{M}$ FITCBSA was used as final concentration for the experiments (prepared in PBS, pH 7.4). The different concentrations of SMZ and SMO drugs were 20,30, 40, 50 and $60 \mu \mathrm{M}$ (prepared in PBS, pH 7.4), used for all binding experiments.

\subsection{Fabrication of microwells}

The lotus leaf surface contains microbumps with height and base diameter in the range of 8-16 $\mu \mathrm{m}$ and 5-10 $\mu \mathrm{m}$, respectively. Soft lithography procedure was used to transfer the topography of lotus leaf on polymeric surfaces to form microwell array. Initially, the lotus leaf was cut into $5 \mathrm{~cm} \times 5 \mathrm{~cm}$ sections and bonded on to glass slides. Then, a PDMS base and curing agent (Sylgard 184, Dow Corning,
USA) were mixed gently with a volume ratio of 10:1 (w/w) and degassed in a desiccator. Thereafter, the mixed volume was poured into the molds and left for $24 \mathrm{~h}$ to allow self-curing without the aid of heating. The cured PDMS surface, was then gently peeled off from the mold. Finally, a negative replica consisting of microwells was formed on the PDMS slabs.

\subsection{Surface modification}

Oxygen plasma treatment was employed to render hydrophilicity to the PDMS microwell-surface to avoid trapping of air bubbles in microwells when the droplet containing proteins comes in contact with the surface. Oxygen plasma treatment introduces polar functional groups such as silanol group ( $\mathrm{SiOH}$ ) on PDMS surfaces thereby changing its surface behavior from hydrophobic to hydrophilic. In the present work, plasma power was kept constant at $70 \mathrm{~W}$ with an oxygen flow rate of $25 \mathrm{sccm}$, for $120 \mathrm{~s}$. The hydrophilicity of the microwell-surface was retained for $3 \mathrm{~h}$, which was well within the experimental duration of drug-protein interaction [21].

\subsection{Instrumentation}

The geometry and dimensions of the microwells have been analyzed by an optical profilometer (Zeta-20, Zeta Instruments, USA) and an environmental scanning electron microscope (ESEM, Philips FEI Quanta-200, The 
Netherlands). The drug-protein interaction in microwells was analyzed by the Fluorescence microscopy (Leica DMi8 microsystems, USA). FT-IR spectra were measured by using Vertex 80 FTIR System (Bruker, Germany) on the microwell platform. All the readings were taken using attenuated total reflection method. For the FTIR study, five samples have been analyzed viz. PBS buffer, BSA, Drug (SMZ, SMO), BSA-drug. The transmittance of buffer solution was subtracted from the spectrum of BSA solution to obtain the peaks of pure BSA. Same method has been applied for BSA-drug transmittance values.

\subsection{Fluorescence study on microwells}

A $2 \mu \mathrm{L}$ droplet of $40 \mu \mathrm{M}$ FITC-BSA was kept over the microwell platform for $5 \mathrm{~min}$ to ensure complete filling of the wells. The excess solution of FITC-BSA was removed by using blotting paper (Whatman ${ }^{\circledR}$ filter Paper, Sigma Aldrich, USA) carefully. First, the fluorescence signal from the microwells was recorded, without adding drug on it. After ensuring the complete filling of microwells, a drop of drug ( $2 \mu \mathrm{L}$ of SMO or SMZ) was added on the microwell surface. The excess drug was removed in the same manner and the fluorescence signal from the microwells of drug-protein interaction was recorded in a time dependent manner using fluorescence microscope (Leica DMi8, USA). All the experiments were conducted in a darkroom to prevent any errors in the data. Initially, a control (only the microwell surface with FITC-BSA) was used to set the parameters of the microscope such as exposure time, gain and intensity. Additionally, it is ensured that no background signal is captured.

\section{Analysis of drug-protein interaction in microwell array}

\subsection{Topography and wettability of microwells}

The 3D optical profilometer measurements revealed that the typical height of the microwells were in the range $8-16 \mu \mathrm{m}$ and opening diameter is in the range $4-8 \mu \mathrm{m}$ as shown in Fig. 2a. Figure $2 \mathrm{~b}$ shows an optical micrograph of microwells on PDMS surface with the corresponding individual microwells characterized. Figure $2 c$ shows scanning electron microscope (SEM) micrograph of a single microwell. It is apparent from these measurements that the typical volume contained by the microwells range from $100 \mathrm{fL}$ to $1 \mathrm{pL}$, which will substantially increase the sensitivity of the fluorescence signal. Figure $2 \mathrm{~d}$ shows the variation of contact angle before and after the oxygen plasma treatment (OPT) on PDMS microwell-surface. As explained earlier, oxygen plasma treatment introduces the polar functional groups on PDMS, thereby changing its surface characteristic from hydrophobic to hydrophillic. The contact angle on as-prepared PDMS microwell-surfaces was measured to be $129.1^{\circ} \pm 3.8^{\circ}$. After the OPT, the contact angle on PDMS microwell-surfaces was reduced to about $\sim 60^{\circ} \pm 2.3^{\circ}$. The surface treatment on PDMSmicrowell surface leads to the complete filling of sample in microwells. The process parameters of OPT were optimized to retain hydrophilicity of PDMS over a long time i.e., $3 \mathrm{~h}$. With the optimal parameters, the change in contact angle on plasma-treated PDMS surface was shown to increase by $12 \%$ (from $60^{\circ}$ to $68^{\circ}$ ) over a period of $3 \mathrm{~h}$ and $68 \%$ (from $60^{\circ}$ to $101^{\circ}$ ) over a period of $6 \mathrm{~h}$. The complete filling of microwells within a minute after placing the sample was confirmed through confocal microscopy measurements [21]. It should be noted that, the experiments to record the fluorescence intensity were conducted within an hour of post-treatment of the microwell-surfaces. In order to understand the distribution and shape of microwells, twostep lithography was conducted to replicate micropillars from a microwell-surface [21]. Figure 2e shows a crosssectional SEM micrograph of micropillars representing the shape of microwells.

\subsection{Fluorescence study of drug-protein interaction}

As explained in Sect. 2, the fluorescence signal from the microwells was recorded and analyzed using fluorescence microscopy. The fluorescence images were acquired from the top surface of the microwells. Another discrete set of experiments was also conducted on plain PDMS surfaces for completeness (not shown). However, the fluorescence signal was observed to weak on plain surfaces as compared to the surfaces with microwells. This observation is in agreement with the previous investigations [18, 21]. The surface with microwells confines FITC-BSA in very small volumes in the range of pico to femtolitres, which leads to highly localized intensification of fluorescence signal. The fluorescence intensity from the microwell array was estimated by ImageJ software (US National Institutes of Health, http://rsb.info.nih.gov/ij/). A total of thirty microwells were considered from each fluorescent micrograph randomly and the average value of the fluorescence intensity was calculated. Typical time-lapse fluorescent micrographs of the FITC-BSA when interacted with different concentrations of SMZ and SMO at three different time intervals are shown in Fig. 3b, c, respectively. As it is evident from the figure, an increase in the concentration of drug on protein leads to a quicker depletion of the fluorescence signal from the microwell array. Further, the quenching of the fluorescence signal with respect to time is also apparent from the figure. A further reduction in fluorescence intensity from the microwell array was not 
Fig. 2 a, b Depth profiles of microwells obtained by a 3D optical profilometer shown along with the corresponding microwells, c SEM image of a single microwell, $\mathbf{d}$ contact angle on microwell surfaces before and after oxygen plasma treatment (OPT), e cross-sectional image of micropillars replicated from a microwell-surface through two-step soft lithography
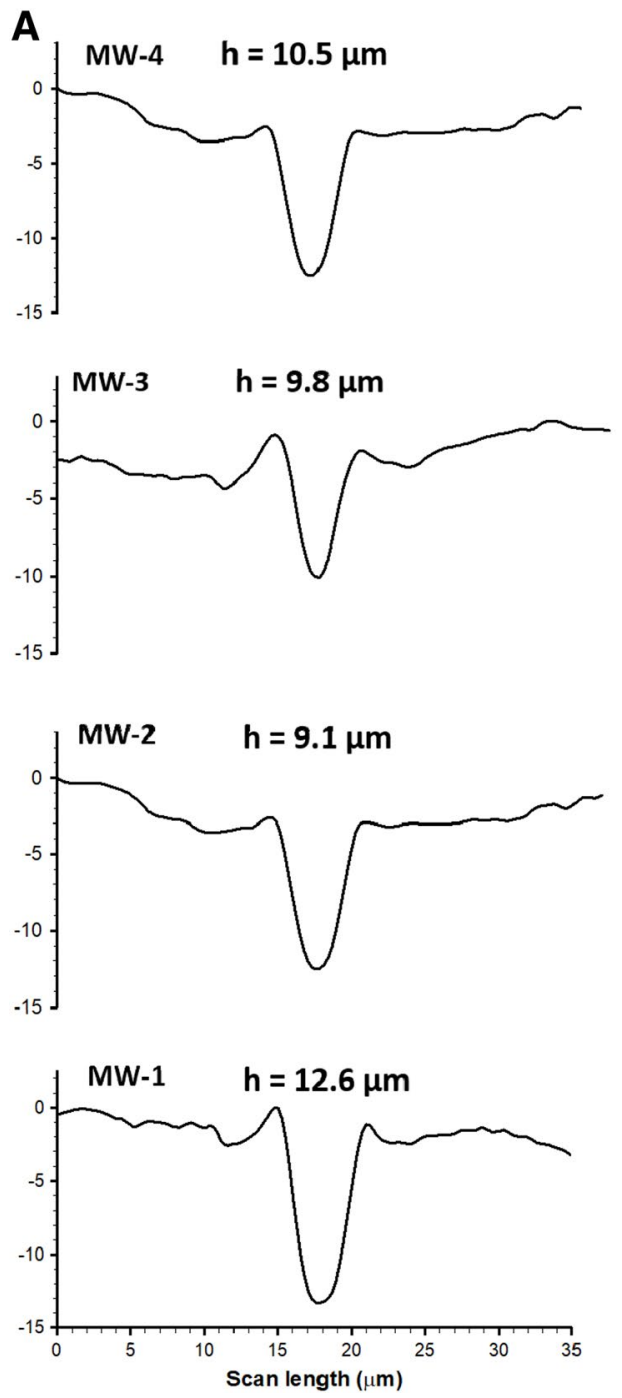

observed beyond $12 \mathrm{~min}$ for both the drug-protein complexes. Also, in Fig. 3c, the control indicates that there is no loss of fluorescence in the absence of the drugs in $11 \mathrm{~min}$. The quenching of fluorescence signal of FITC-BSA in the presence of SMO and SMZ is further quantified as shown in Fig. $4 a$, b, respectively. Here, the fluorescence intensity from the microwell array after the formation of the drug-protein complex is normalized by the fluorescence intensity from the microwell of the pristine FITC-BSA. The base line in these figures indicates variation of the fluorescence intensity of the pristine FITC-BSA as a function of time. It is clear from the figure that, the quenching is a function of time at low concentration of the drug, while fluorescence signal quenches rapidly at higher concentrations. Fluorescence quenching can either occur through dynamic quenching mode or static quenching mode. In order to study the quenching mechanism of BSA in the presence of SMZ and SMO, the Stern-Volmer equation is used which is given by
B

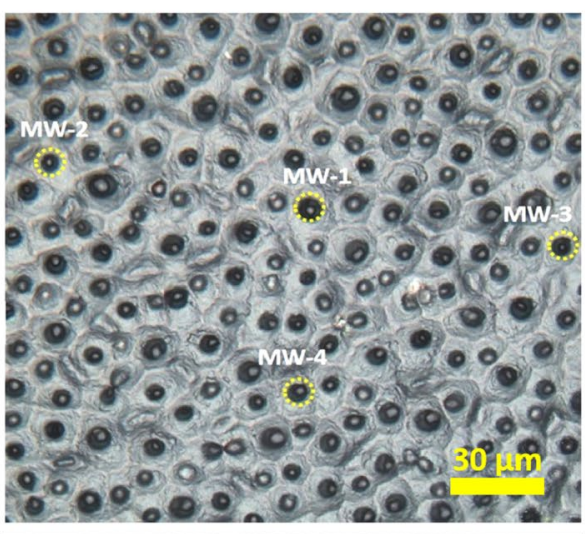

C

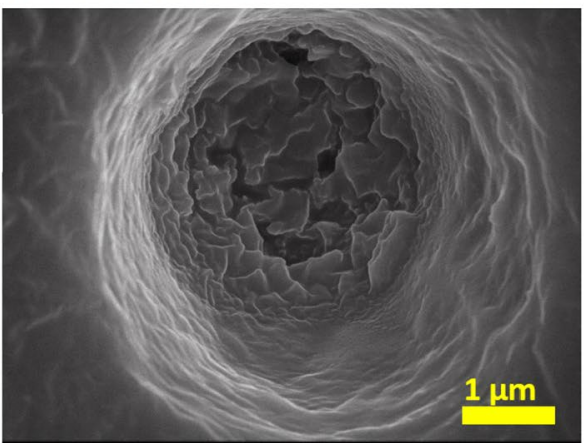

D

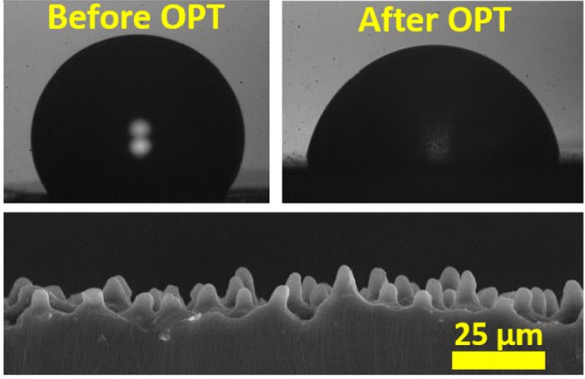

$\frac{F_{0}}{F}=1+K_{S V}[Q]$

where $F$ and $F_{0}$ are the fluorescence intensity of BSA with and without quencher (drug), $K_{s v}$ and $Q$ are quenching constant and concentration of quencher, respectively.

In the case of static quenching interaction, small molecule binds to the active sites of macromolecule and the binding constant $K a$ and binding number $(\mathrm{n})$ is determined by equation

$\log \left(\frac{F_{0}-F}{F}\right)=\log K_{a}+n \log [Q]$

The Stern-Volmer plots of BSA in the presence of different concentrations of SMO and SMZ are shown in Fig. $5 a$, b. As can be seen, a linear relationship is exhibited by the fluorescence intensity with the concentration of the drug. The values of $K_{s v^{\prime}}$ can be determined from the slope of curve of $F 0 / F$ versus $Q$ curves from Eq. 1. Similarly, the values of 
A
$1 \mathrm{~min}$.

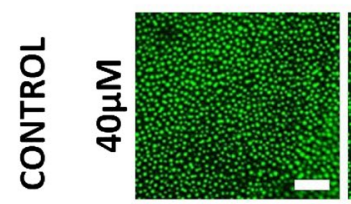

B

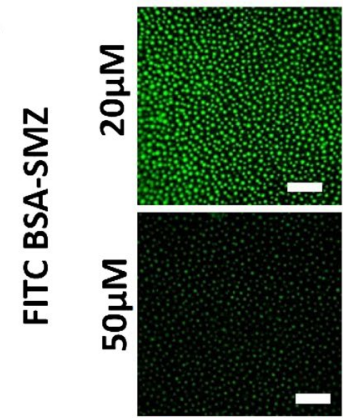

C

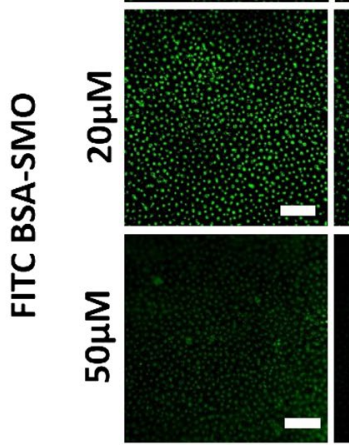

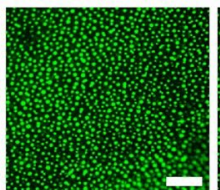
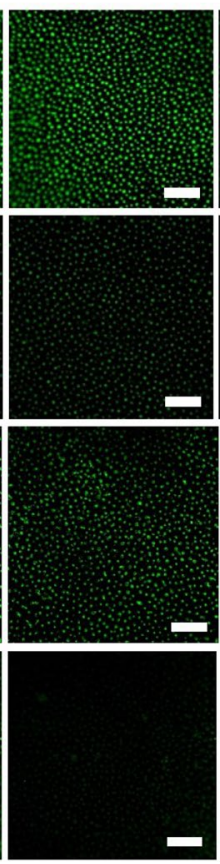

$5 \mathrm{~min}$.
$11 \mathrm{~min}$.
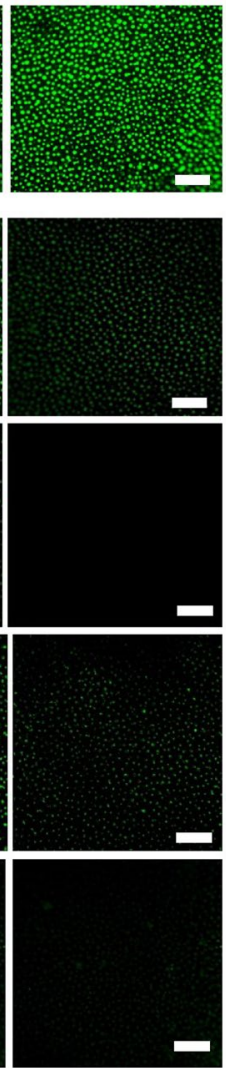

Fig. 3 The fluorescence micrographs of FITC-BSA in biomimetic microwell in the absence and presence of drugs. a No loss of fluorescence intensity in FITC-BSA at different time intervals without drug (scale bar-30 $\mu \mathrm{m}$ ). Variation of fluorescence intensity of FITCBSA (b) with SMZ drug, and $\mathbf{c}$ with SMO drug for different concentrations at different time intervals (scale bar $-30 \mu \mathrm{m}$ )
$K_{a}$ and $n$ can be evaluated from the slope and intercept of $\log (F 0-F) / F$ versus log $[Q]$, using Eq. 2 . The estimated values of these constants are compared with the previously reported Fluorescence spectroscopy method as listed in Table 1 . The value of binding constant $(n)$ approximately equal to unity, indicating that there is one binding site in BSA for SMO and SMZ for interaction. As it can be seen, a reasonably good agreement is found between the microwell-based method and bulk method [19]. Furthermore, the timescale of decay in fluorescent intensity of drug in the present study is also in agreement with the bulk method $[20,22]$. This indicates that the present method can be used to study the kinetics of the interaction between drug and ligand.

\subsection{Molecular docking}

Molecular docking was employed to understand the active sites and forces responsible for interaction between drug and protein. Figure $6 \mathrm{a}$ shows the ribbon structure of BSA with active sites: namely I A and II B. Figure $6 \mathrm{~b}$ shows the chemical structure of $S M Z$ and $S M O$, used for molecular docking. The structure of BSA (4F5S) was obtained from the protein data bank (http://www. rcsb.org/pdb). For the docking analysis, essential hydrogen bonds, Kollman charges were added to the protein and water molecules were removed using Auto Dock Vina tool. In drug molecule (SMZ, SMO), MMFF94 force has been used for energy of minimization [23]. Gasteiger partial charges were added and rotatable bonds were defined using Avogadro tool. The grid box was fixed with $13 \times 16 \times 124$ with a grid spacing of $0.375 \AA$. In the
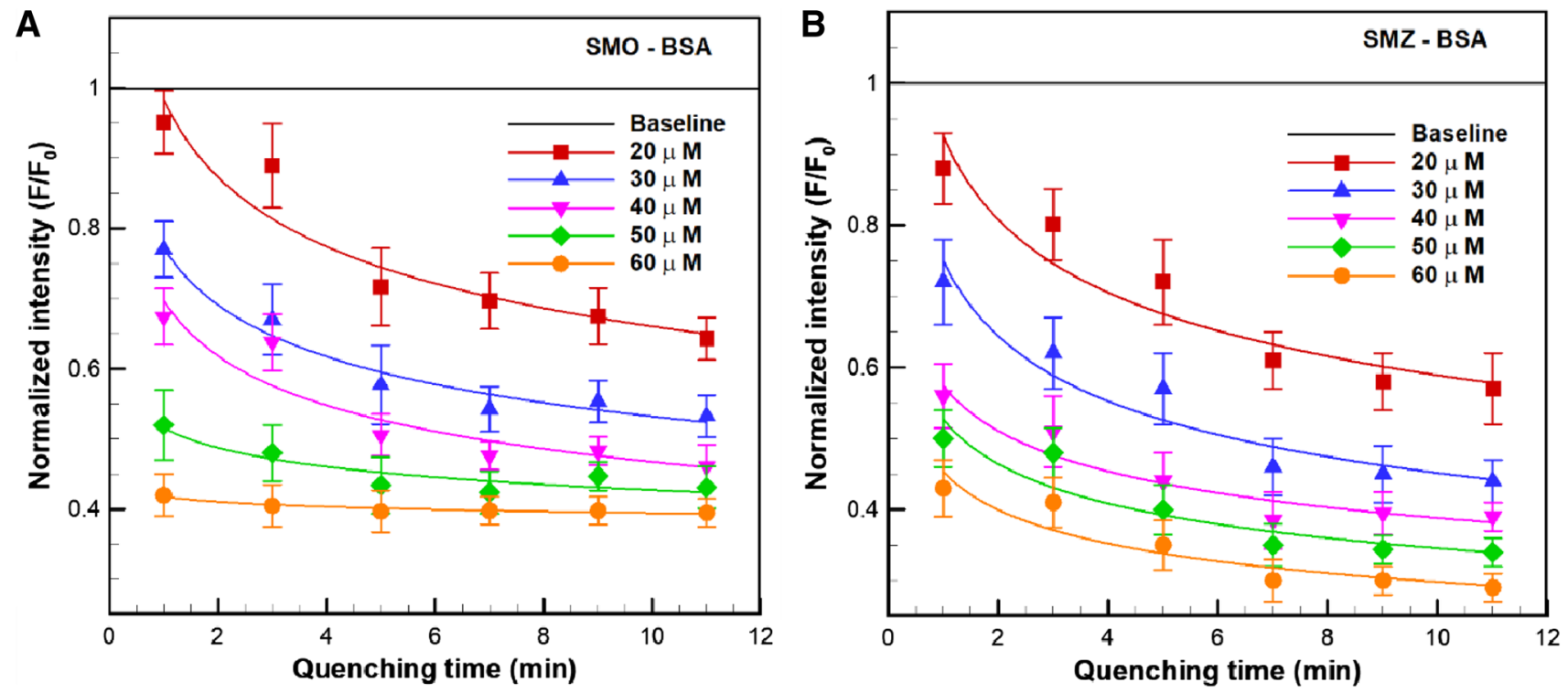

Fig. 4 A variation of normalized fluorescent intensity with quenching time of BSA with a SMO and b SMZ drugs

\section{SN Applied Sciences}



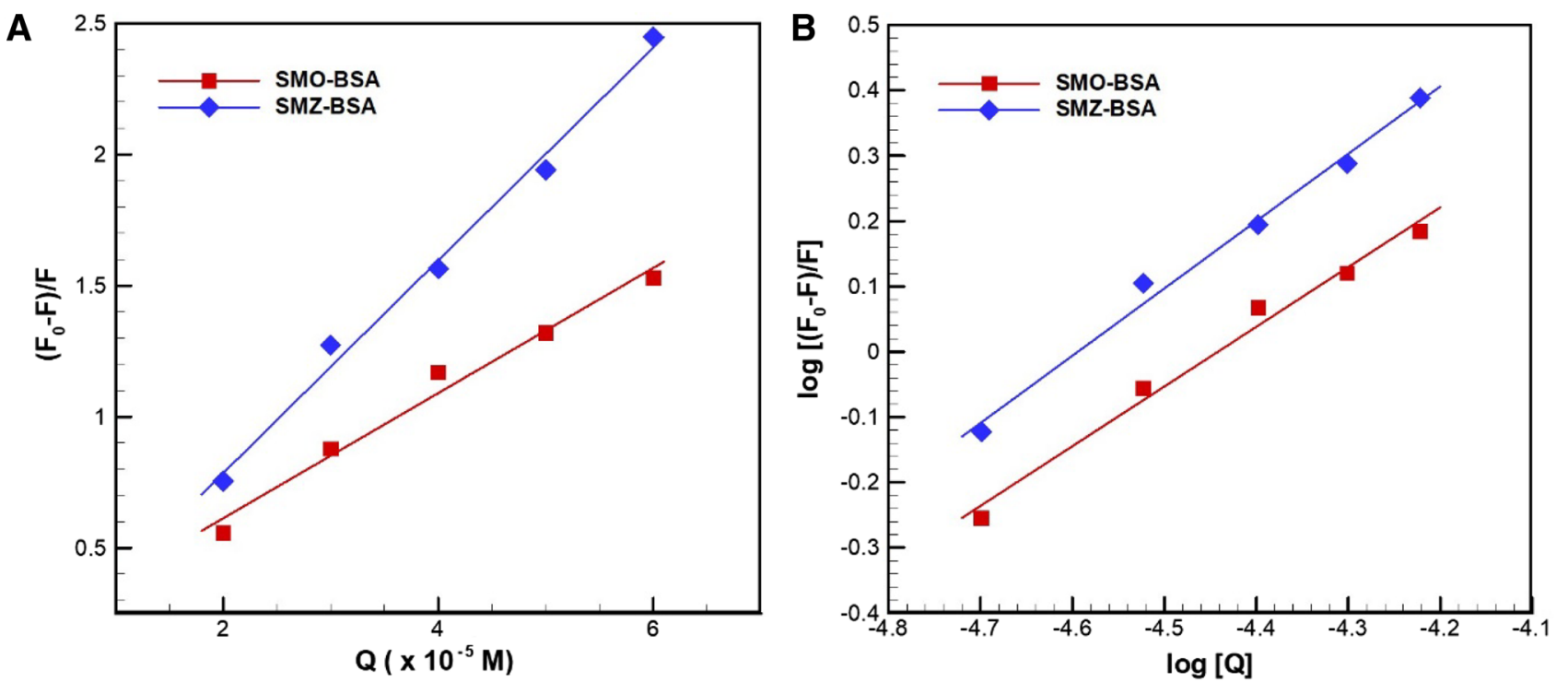

Fig. 5 a Stern-Volmer plots for quenching of BSA with SMZ and SMO (b) Plot of log $(F 0-F) / F$ versus log $[Q]$ for BSA with SMZ and SMO

Table 1 Stern-Volmer quenching constant $\left(K_{s v}\right)$ and Stern-Volmer association constant $\left(K_{a}\right)$ of BSA with SMZ and SMO

\begin{tabular}{|c|c|c|c|c|c|c|c|c|}
\hline \multirow[t]{2}{*}{ Complex } & \multicolumn{2}{|c|}{$K_{s v}\left(10^{4} \mathrm{M}^{-1}\right)$} & \multicolumn{2}{|c|}{$K_{a}\left(10^{4} \mathrm{M}^{-1}\right)$} & \multicolumn{2}{|l|}{$\mathrm{n}$} & \multicolumn{2}{|l|}{$R^{a}$} \\
\hline & MW & SP & MW & SP & MW & SP & $\mathrm{K}_{\mathrm{sv}}$ & $\mathrm{K}_{\mathrm{a}}$ \\
\hline SMO-BSA & 0.34 & 0.51 & 1.108 & 0.618 & 0.96 & 0.99 & 0.982 & 0.992 \\
\hline SMZ-BSA & 0.52 & 0.76 & 1.563 & 0.911 & 0.93 & 1.03 & 0.975 & 0.995 \\
\hline
\end{tabular}

$M W$ microwell platform, SP spectroscopy method [19], $\mathrm{R}^{\mathrm{a}}$ linear correlation coefficient end, docking simulation was performed using Lamarckian genetic algorithm. PyMOL and Ligplot frameworks have been employed to analyze the simulation result. It has been found that PHE506, PHE508, LYS524, ALA527, LEU528, LEU531, VAL546, PHE550, LEU574, THR578 were the most vital residues for SMZ and BSA interaction. In the same manner, THR190, SER192, ALA193, ARG196, ARG435, TYR451, LEU454, ARG458 were the active residues for SMO-BSA interaction. It can be seen that Fig. 7a, $c$ and Table 2 showed the best possible interacting model and the residues of BSA involved in the interaction. The most favorable conformation of docked pose in Fig. 7a, c, revealed the binding of drug with protein. The docking results shows that hydrophobic interaction are the main forces for the drug-protein complex formation. Furthermore, in Fig. 7b, the two nitrogen atom N1 and $\mathrm{N} 2$ form two $\mathrm{H}$-bond with the $\mathrm{OH}$-bond of THR-190 and -NH of ARG-458 respectively. In the same manner, $\mathrm{H}$-bonding occurred in SMZ drug as shown in Fig. $7 d$. From the docking analysis, we can infer that hydrophobic and $\mathrm{H}$-bonding was responsible for the interaction of drug with protein.

\subsection{FT-IR spectroscopic measurements}

FT-IR spectra of protein exhibited the various amide bonds due to the different vibrations of peptide. Among all amide bonds, amide I is more sensitive to interaction than amide II. Therefore, amide I bond is the more useful bond to study the interaction of drug and protein [23]. The amide I and II peaks occur in the region of $1700-1600 \mathrm{~cm}^{-1}$ and $1600-1500 \mathrm{~cm}^{-1}$ respectively (as shown in Fig. 8a, b). Figure $8 a$, b shows the spectra of SMO \& SMZ free BSA and bound BSA with different infrared spectrum. In Fig. $8 a$, b, the peaks shows that there is a noticeable shift of amide I in both the result after addition of drugs (SMZ \& SMO). After the interaction with SMO and SMZ, the peak position of amide I in the infrared spectrum has been changed from 1653.28 to $1645.98 \mathrm{~cm}^{-1}$ and $1656.59 \mathrm{~cm}^{-1}$ to $1650.71 \mathrm{~cm}^{-1}$. These results indicate that both the drugs interacted with BSA. 
A

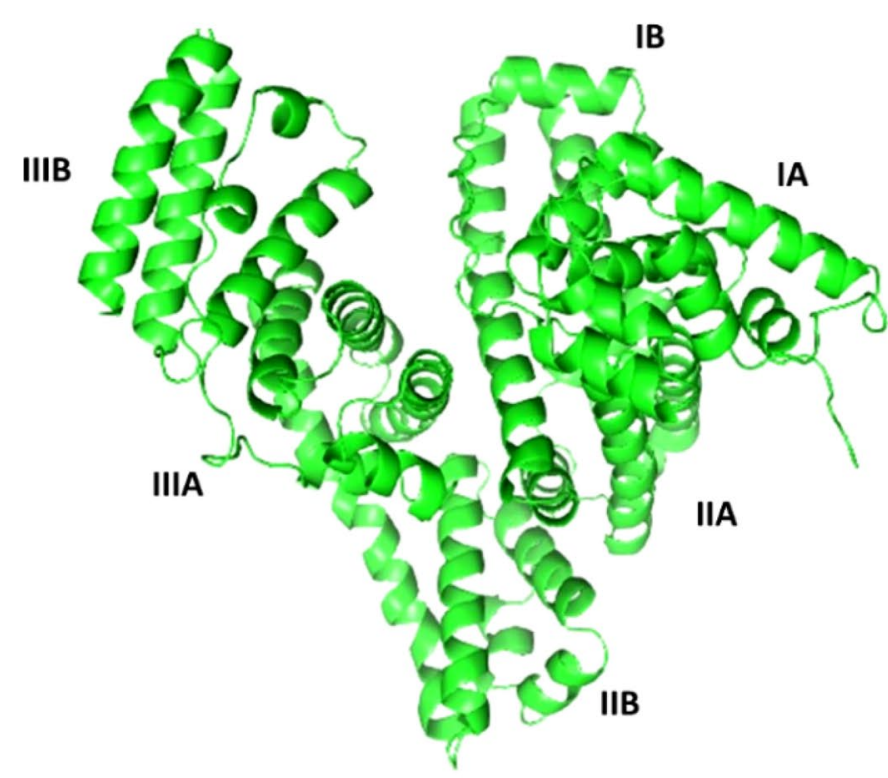

B
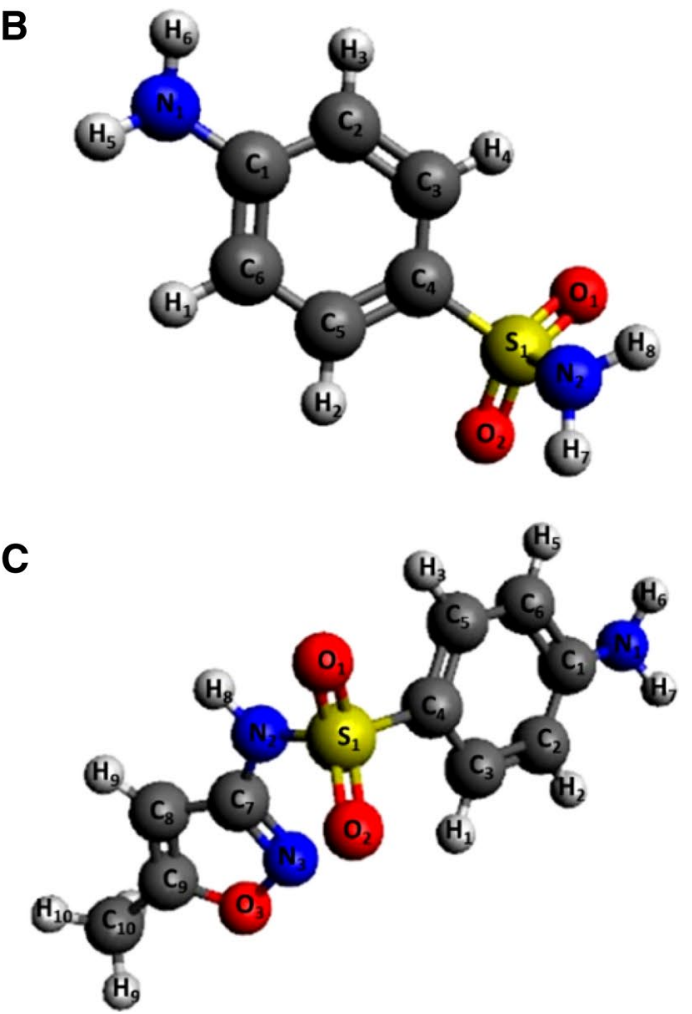

Fig. 6 a Ribbon presentation of 4F9S (BSA) drug with different binding sites, b chemical structures of sulphanilamide (SMO) and c sulfamethoxazole (SMZ)

\section{Integration of microwell platform to microfluidic device}

Microfluidic technologies help in achieving high-throughput sampling, parallelization and automation, which lead to development costs in drug discovery at affordable levels. Among all the microfluidic methods, droplet microfluidics has shown a great promise in many applications related to biochemical assays due to its ability to precisely control very small volumes of samples. Additionally, the droplet dispensing and removal from open platforms is time consuming and involves sample wastage. For instance, once the droplet is dispensed on the microwell surface, it requires time to equilibrate over the surface based on the intrinsic wettability of the substrate. Once complete filling takes place, the additional volume of the droplet was removed from the surface. This step could lead to partial removal of the sample from the microwells. On the other hand, in closed platform, the receding meniscus in conjunction with capillary forces on the top plate aid in capturing the right amount of sample. Subsequently, the additional volume of the droplet can be employed to pattern the sample in microwells over large areas. Furthermore, multiple drug libraries can be investigated on a single microwell platform. To this end, we have incorporated the microwell platform into a magnetic digital microfluidic device recently reported by our group, to investigate the fluorescence quenching of drug-protein complex [24]. Briefly, the device consists of top and bottom plates containing microwells and micropillars, respectively as shown in Fig. 9a.

The magnetic liquid marbles containing either drug or protein were encapsulated by magnetic nanoparticles and driven by a permanent magnet in the chamber formed by the top and bottom plates. The microwells on the top plate capture the biological entities present in the magnetic liquid marble through the capillary wicking mechanism, while micropillars on the bottom plate facilitate smooth movement of the magnetic liquid marble. In order to investigate the binding of the drug with the protein, initially a magnetic liquid marble $(\sim 2 \mu \mathrm{l})$ containing the FITC-BSA was introduced through the inlet. The concentration of the FITC-BSA in the magnetic liquid marble was 40 $\mu \mathrm{M}$. Subsequently, the magnetic liquid marble was driven by the permanent magnet back and forth in the chamber to facilitate the imbibition of microwells by FITC-BSA as shown in Fig. 9a.

Once the FITC-BSA was captured in the microwell array on the top plate, the magnetic liquid marble was removed from the outlet and fluorescence imaging was 

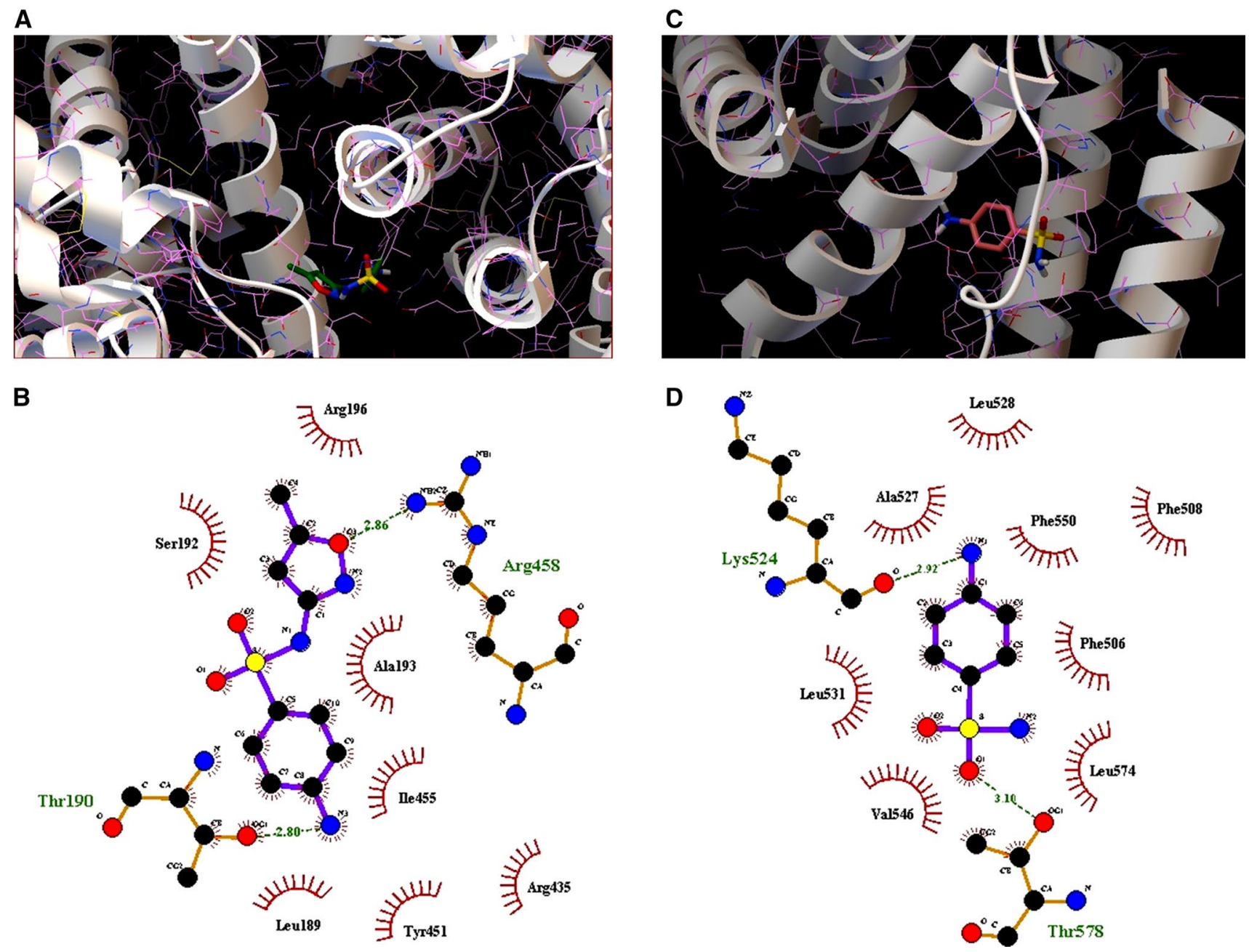

Fig. 7 Binding mode and conformation of a SMO, c SMZ docked to BSA. The residues of BSA are represented by lines and ligand is represented by ball and sticks. $\mathbf{b}, \mathbf{d} 2 \mathrm{D}$ representation of hydrogen

bonding of interaction of BSA with SMO \& SMZ. The green dashed line depicted the $\mathrm{H}$-bonding

Table 2 Molecular docking parameter of BSA with SMZ and SMO interaction

\begin{tabular}{llll}
\hline & Amino acid residues & $\begin{array}{c}\text { Est. free energy of } \\
\text { binding (Kcal/mol) }\end{array}$ & Forces involved \\
\hline SMZ & $\begin{array}{l}\text { PHE506, PHE508, LYS524, ALA527, LEU528, LEU531, VAL546, PHE550, } \\
\text { LEU574, THR578 }\end{array}$ & -6.9 & Hydrophobic interaction \& H-bonding \\
SMO & THR190, SER192, ALA193, ARG196, ARG435, TYR451, LEU454, ARG458 & -5.9 & Hydrophobic interaction \& H-bonding \\
\hline
\end{tabular}

conducted. Thereafter, magnetic liquid marbles containing either SMZ were pipetted into the chamber through the inlet. In a similar fashion as mentioned above, the drug was allowed to interact with the FITC-BSA prefilled microwells. The concentration of the drugs was chosen at two levels i.e., $20 \mu \mathrm{M}$ and $50 \mu \mathrm{M}$. Figure $9 \mathrm{~b}$, c shows the fluorescence micrographs of the pristine FITC-BSA and SMZ-BSA complex, respectively for a drug concentration of $20 \mu \mathrm{M}$. Similarly, Fig. 9d, e shows the fluorescence micrographs for a drug concentration of $50 \mu \mathrm{M}$. It is apparent from these figures that the fluorescence quenching occurred due to the interaction between the SMZ and BSA. These results show the prospective application of microwell-integrated microfluidic platforms in the early stages of drug discovery. For instance, the sample volume required in fluorescence spectroscopy is typically in the range of $1.5-3.5 \mathrm{~mL}$ (contained by cuvettes) can be significantly reduced by thousand-fold in the magnetic digital microfluidic device 

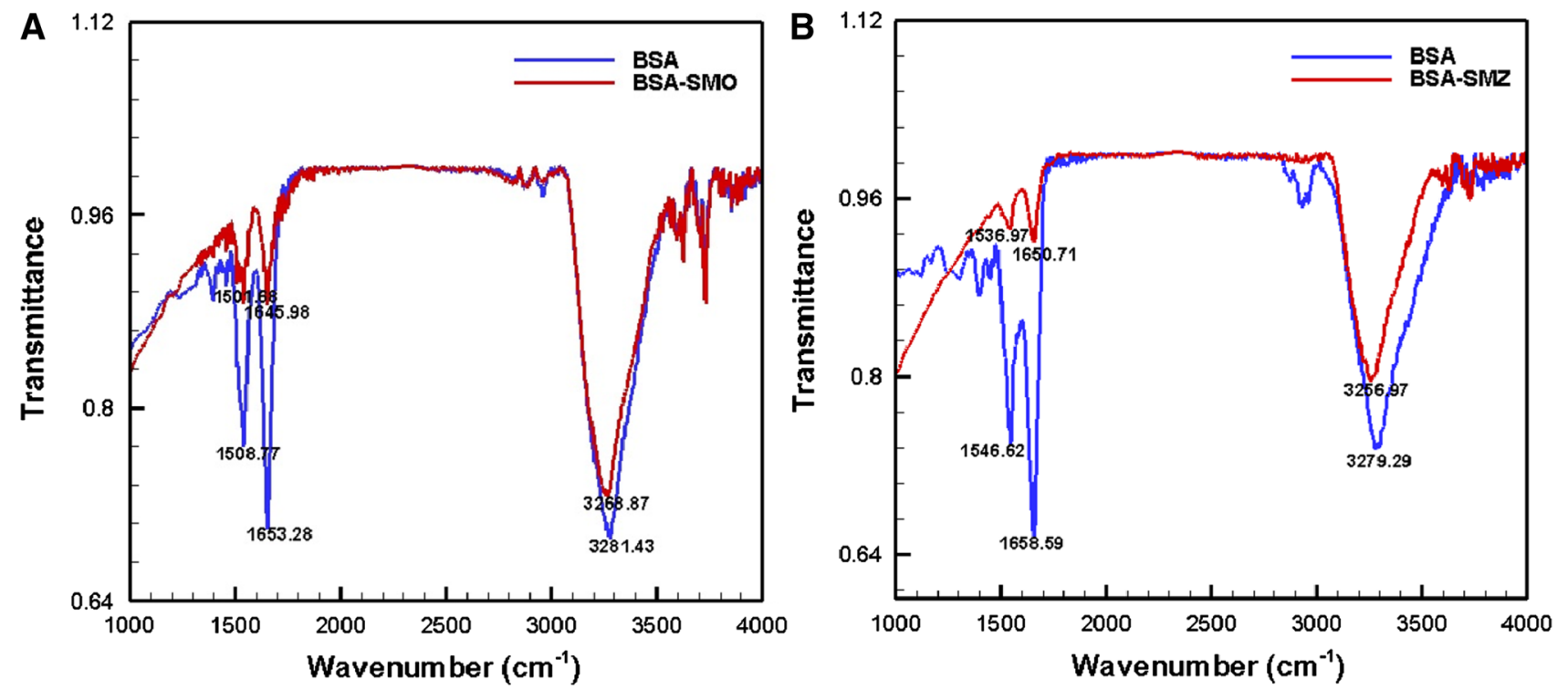

Fig. 8 a, b FT-IR spectrum of BSA after subtracting the spectrum of buffer \& Infrared Spectrum of bound BSA (after addition of drug)

A

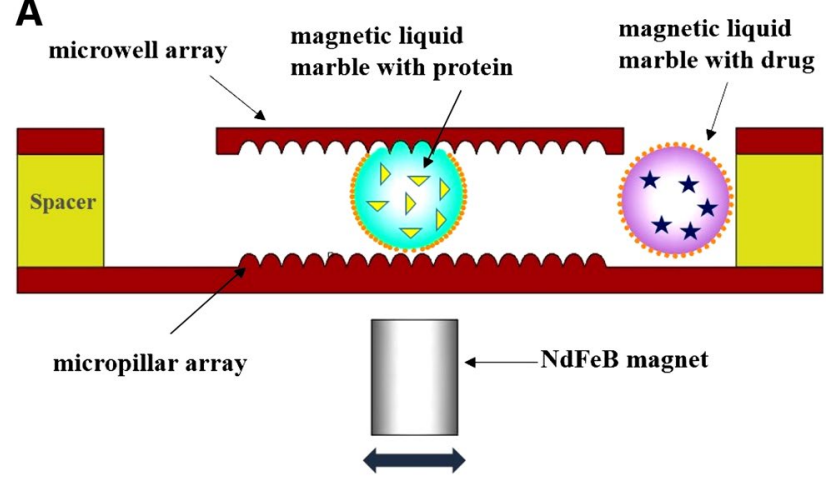

B
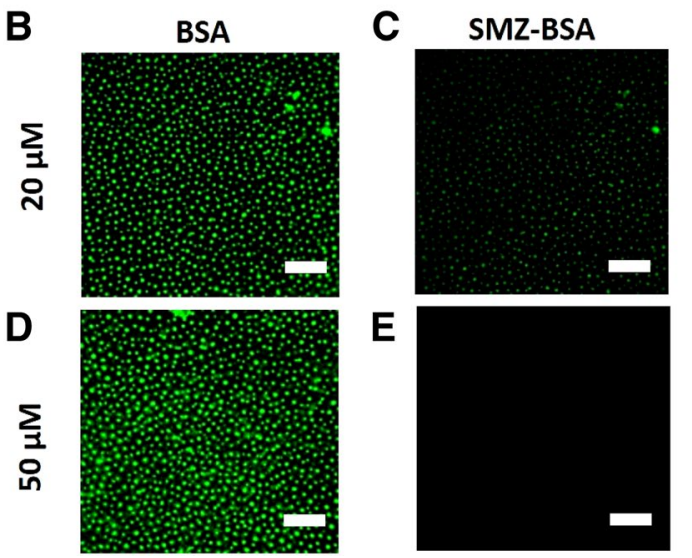

Fig. 9 a A schematic of magnetic actuated, biomimetic microdevice for drug-protein interaction. b, c Fluorescence micrograph of FITC-BSA and SMZ-BSA complex (after $7 \mathrm{~min}$ ) at concentration of $20 \mu \mathrm{M}(\mathbf{d}, \mathbf{e})$ at concentration of $40 \mu \mathrm{M}$ (scale bar-30 $\mu \mathrm{m}$ )
( $2 \mu \mathrm{L}$ per magnetic liquid marble). Subsequently, the total cost of trials will be substantially reduced with a parallel increase in experiments required to meet strict reproducibility standards during the pre-clinical stages.

\section{Summary}

We have demonstrated fluorescence quenching of drug-protein bound complex on a bio-inspired microwell platform to characterize the binding of antibacterial drugs such as SMO and SMZ with BSA. To the best of our knowledge, we have for the first time reported the visualization of fluorescence quenching of protein in the presence of drug on a microwell array. The Stern-Volmer analysis of the fluorescence signal obtained from the microwells provided crucial parameters of the drug-protein interaction. Furthermore, the evaluated constants of the binding of SMO and SMZ with the BSA are well comparable with the fluorescence spectroscopy. The usage of microwells fabricated from the naturally occurring lotus leaf molds reduced the cost and the number of steps involved in fabrication.

The proposed method can be easily implemented with continuous flow or digital microfluidics-based devices. When used with such microfluidic systems the present method to investigate the drug-protein interaction in microwells has the potential to find application in binding assays and kinetic studies and can replace a few of the bulk methods. We envisage that the encapsulation of different drug libraries in discrete droplets and investigating the interaction of drugs with protein prefilled-microwells through EWOD (electrowetting-on-dielectric) or magnetic

\section{SN Applied Sciences}


digital microfluidics-based devices will enable to discover the full potential of the present method.

Acknowledgements We are grateful to Sophisticated Analytical Instrumentation Facility (SAIF), IIT Bombay for allowing us to access FT-IR facility.

\section{Compliance with ethical standards}

Conflict of interest On behalf of all authors, the corresponding author states that there is no conflict of interest.

\section{References}

1. Keserü GM, Makara GM (2006) Hit discovery and hit-to-lead approaches. Drug Discov today 11(15-16):741-748

2. Davis AM, Keeling DJ, Steele J, Tomkinson NP, Tinker AC (2005) Components of successful lead generation. Curr Top Med Chem 5(4):421-439

3. Olson RE, Christ DD (1996) Plasma protein binding of drugs. In: Bristol JA (ed) Annual reports in medicinal chemistry, vol 31. Academic Press, New York, pp 327-336

4. Banker MJ, ClarkTH, Williams JA (2003) Development and validation of a 96-well equilibrium dialysis apparatus for measuring plasma protein binding. J Pharm Sci 92(5):967-974

5. Judd RL, Pesce AJ (1982) Free drug concentrations are constant in serial fractions of plasma ultrafiltrate. Clin Chem 28(7):1726-1727

6. Kurz H, Trunk H, Weitz B (1977) Evaluation of methods to determine protein-binding of drugs. Equilibrium dialysis, ultrafiltration, ultracentrifugation, gel filtration. Arzneimittel-Forschung 27(7):1373-1380

7. Hage DS (2002) High-performance affinity chromatography: a powerful tool for studying serum protein binding. J Chromatogr B 768(1):3-30

8. Pedersen JT, Østergaard J, Houen G, Heegaard NH (2008) Affinity capillary electrophoresis for identification and investigation of human Gc-globulin (vitamin D-binding protein) and its isoforms interacting with G-actin. Electrophoresis 29(8):1723-1733

9. Song Y, Liu Y, Liu W, Villamena FA, Zweier JL (2014) Characterization of the binding of the Finland trityl radical with bovine serum albumin. RSC Adv 4(88):47649-47656

10. Naik PN, Chimatadar SA, Nandibewoor ST (2009) Study on the interaction between antibacterial drug and bovine serum albumin: a spectroscopic approach. Spectrochim Acta Part A Mol Biomol Spectrosc 73(5):841-845
11. Li X, Wang G, Chen D, Lu Y (2014) Interaction of procyanidin B3 with bovine serum albumin. Rsc Adv 4(14):7301-7312

12. Wang $X, X u$ J, Liu C, Chen $Y$ (2016) Specific interaction of platinated DNA and proteins by surface plasmon resonance imaging. RSC Adv 6(26):21900-21906

13. Yang $X$, Wang $Y$, Wang $K$, Wang $Q$, Wang $P$, Lin $M$, Chen $N$, Tan $Y$ (2014) DNA aptamer-based surface plasmon resonance sensing of human C-reactive protein. RSC Adv 4(58):30934-30937

14. Walt DR (2014) Protein measurements in microwells. Lab Chip 14(17):3195-3200

15. $\mathrm{Ng} \mathrm{AH}, \mathrm{Chamberlain} M D$, Situ H, Lee V, Wheeler AR (2015) Digital microfluidic immunocytochemistry in single cells. Nat Commun 6:7513

16. Kim YT, Choi JY, Chen Y, Seo TS (2013) Integrated slidable and valveless polymerase chain reaction-capillary electrophoresis microdevice for pathogen detection. RSC Adv 3(22):8461-8467

17. Lombardi D, Dittrich PS (2011) Droplet microfluidics with magnetic beads: a new tool to investigate drug-protein interactions. Anal Bioanal Chem 399(1):347-352

18. Wang T, Zhang M, Dreher DD, Zeng Y (2013) Ultrasensitive microfluidic solid-phase ELISA using an actuatable microwellpatterned PDMS chip. Lab Chip 13(21):4190-4197

19. Rajendiran N, Thulasidhasan J (2015) Interaction of sulfanilamide and sulfamethoxazole with bovine serum albumin and adenine: spectroscopic and molecular docking investigations. Spectrochim Acta Part A Mol Biomol Spectrosc 144:183-191

20. Meyer-Almes FJ (2015) Kinetic binding assays for the analysis of protein-ligand interactions. Drug Discov Today Technol 17:1-8

21. Sharma H, John K, Gaddam A, Navalkar A, Maji SK, Agrawal A (2018) A magnet-actuated biomimetic device for isolating biological entities in microwells. Sci Rep 8(12717):1-14

22. Paul BK, Ray D, Guchhait N (2013) Unravelling the binding interaction and kinetics of a prospective anti-HIV drug with a model transport protein: results and challenges. Phys Chem Chem Phys 15(4):1275-1287

23. Naik PN, Nandibewoor ST, Chimatadar SA (2015) Non-covalent binding analysis of sulfamethoxazole to human serum albumin: fluorescence spectroscopy, UV-vis, FT-IR, voltammetric and molecular modeling. J Pharm Anal 5(3):143-152

24. Luo J, Luo J, Yuan C, Zhang W, Li J, Gao Q, Chen H (2015) An eco-friendly wood adhesive from soy protein and lignin: performance properties. RSC Adv 5(122):100849-100855

Publisher's Note Springer Nature remains neutral with regard to jurisdictional claims in published maps and institutional affiliations. 